\title{
SPONTANEOUS SYMMETRY BREAKING IN QUASI-SUPER-RENORMALIZABLE MODELS
}

\author{
Martin B EINHORN and Gary GOLDBERG \\ Rundall Lahoraton of Phystcs, Unuersity of Michigan, Ann Arbor, MI, USA
}

Received 11 September 1986

\begin{abstract}
By studying the effective potential and renormalization group for quasi-super-renormalizable models it is demonstrated the models can undergo spontaneous symmetry breaking in a manner consistent with stability A relation among ratios of masses is seen to hold at the minimum of the potential
\end{abstract}

\section{Introduction}

In a recent paper [1] we introduced the notion of quasi-super-renormalizable theories as those models obtained from finite, globally supersymmetric theories by adding arbitrary soft-breakıng terms (scalar masses, fermıon masses, and cubic scalar couplings) The gauge coupling constant is not renormalized in such models, while the massive parameters in general require infinite counterterms, the soft breakings can be chosen to leave the theories finite, but as these finiteness relations are not enforced by any known symmetry, such a choice, we argued, would involve an unnatural fine tuning of parameters For example, one could take any field theory which has a fixed point and choose the coupling constant to take on precisely the value at that point. whereupon the theory has a zero beta function, but one would not imagine one had a sensible finite theory, as a slight deviation from this metastable value would cause the parameter to run with the scale In ref [1] we showed that the finiteness relations among the soft breakings correspond to an infrared attractive hypersurface in the space of the coupling constants and masses. and that a small deviation from finiteness grows with energy so that in the ultraviolet limit there is no memory of these relations Such globally supersymmetric theories are therefore unappealing as prototypes of a fundamental theory that would be expected to be finite in the high energy regime But they do have an interesting feature since an arbitrary set of soft breakings tends to converge toward the fixed hypersurface in the infrared, relations among masses - in particular, between boson and fermion masses - may emerge naturally in the low-energy effective field theory 
[2] Thus quasi-super-renormalizable models offer intriguing possibılıties for model building

In this paper we discuss the effective potential for quası-super-renormalizable models Spontaneous symmetry breaking (SSB) does not occur in the models which are arranged to be finite [6] - in certain directions the potential due to cubic and quartic terms is flat, so broken symmetry states can be degenerate with the unbroken phase Adding negative masses-squared, the usual signature of spontaneous symmetry breaking, causes the potential to be unbounded from below. destabilizing the theory But in non-finite models the mass parameters run with the scale. and a scalar mass-squared may change sign, engenderıng symmetry breakıng without destabilization As in softly-broken supergravity models [3] the mass could be positive in the ultraviolet (stabilizing the ground state) but negative in the infrared (driving spontaneous breakıng of the gauge symmetry)

We first discuss, in the next section. the one-loop contribution to the effective potential As with the Coleman-Weinberg analysis of SSB bv radiative corrections [4], this perturbative analysis suffices to establish the phenomenon when the normalization scale is chosen appropriately In sect 3 we consider the renormalization-group-improved effective potential and demonstrate that SSB is compatible with stability, in fact, the effective potential grows at large scales Finally, in sect 4. we conclude with a summary of the possible role for quasi-super-renormalizable models

\section{The effective potential}

The model we are studying is the softly broken $N=4$ supersymmetric Yang-Mills theory

$$
\begin{aligned}
L= & -\frac{1}{4} F_{\mu \nu} F^{\mu v}+\frac{1}{2}\left(D_{\mu} A_{i}\right)\left(D^{\mu} A^{\prime}\right)+\frac{1}{2}\left(D_{\mu} B_{l}\right)\left(D^{\mu} B^{\prime}\right)+\frac{1}{2} \bar{\lambda}_{k} D \lambda_{h} \\
& -\frac{1}{2} \bar{\lambda}_{K}\left(m_{\mathrm{f}}\right)_{K I} \lambda_{I}+\frac{1}{2} \lg \bar{\lambda}_{K}\left[\Gamma_{\lambda I}^{\prime} \phi^{\prime} \lambda_{I}\right]-V_{0}
\end{aligned}
$$

A trace over gauge indices is implied The theory contains six spin-zero fields, four Majorana fermions, and one massless gauge boson All fields are in the adjoint representation of the arbitrary gauge group $A^{\prime}=A^{a} T^{a} \cdot\left[T^{a}, T^{h}\right]=\imath f^{a h c} T^{c} . \operatorname{Tr} T^{a} T^{b}$ $=C_{2} \delta^{a h}$ We can write the potential as

$$
V_{0}=\frac{1}{2} m_{l j}^{2} \phi_{l} \phi_{l}+\frac{l}{31} c_{k j k} \phi_{l}\left[\phi_{J} \cdot \phi_{k}\right]-\frac{1}{4} g^{2}\left[\phi_{l}, \phi_{l}\right]^{2}
$$


Here, $\iota, J, k=1,2, \quad 6$ and $K, L=1, \quad, 4$, are indices for an erstwhile SU(4) or $\mathrm{O}(6)$ global symmetry ${ }^{\star}$ imposed by supersymmetry but broken by eq (2.2)

The effective potential is, to one-loop order and in the Landau gauge,

$$
V_{\text {eff }}=V_{0}+V_{1},
$$

where

$$
V_{1}=\frac{1}{64 \pi^{2}} \operatorname{Str} M^{4} \ln \frac{M^{2}}{\mu^{2}}
$$

The supertrace, Str, 1 s the sum over bosons minus the sum over fermıons, weighted by the number of helicity states $2 J+1$ This form of the effective potential assumes a renormalization scheme where polynomial terms are absorbed into the counterterms, and one should subtract from it the value it takes when all coupling constants vanish (so $V_{1}$ is zero for a free theory) The vector. fermion, and scalar mass matrices are

$$
\begin{aligned}
& \left(M_{\mathrm{V}}^{2}\right)^{a b}=g^{2} f^{a d e} f^{b c e} \phi_{k}^{d} \phi_{k}^{c}, \\
& \left(M_{\mathrm{F}}^{+} M_{\mathrm{F}}\right)_{K L \alpha \beta}^{a b}=\left(m_{\mathrm{f}}^{2}\right)_{K L} \delta^{a b} \delta_{\alpha \beta}-g f^{a b c} \phi_{l}^{c}\left(m_{\mathrm{f}} \Gamma^{l}+\Gamma^{++} m_{f}\right)_{K L \alpha \beta} \\
& +g^{2} f^{a c e} f^{h d e} \phi_{\imath}^{c} \phi_{J}^{d d}\left(\Gamma^{t+} \Gamma^{J}\right)_{K L \quad \alpha \beta}, \\
& \left(M_{\mathrm{S}}^{2}\right)_{i j}^{a b}=m_{i j}^{2} \delta^{a b}-c_{t \jmath h} f^{a b c} \phi_{h}^{\iota}+g^{2}\left\{f^{a c e} f^{h d e}\left[\phi_{k}^{c} \phi_{h}^{d} \delta_{l,}-\phi_{l}^{\iota} \phi_{j}^{d}\right]\right. \\
& \left.+f^{a b e} f^{c d t} \phi_{l}^{c} \phi_{l}^{d}\right\}
\end{aligned}
$$

(In eq (2 3) the trace over Dirac indices has already been factored out)

We are interested in the flat directions of the tree potential, in the absence of soft breakings, $m^{2}=m_{f}=c_{\imath \jmath k}=0$, the potential $V_{0}$ is zero only when $\left[\phi_{t}, \phi_{j}\right]=0$, $1 \mathrm{e}$ when the fields are in the Cartan subalgebra of the group In a flat direction, therefore, the fields $\phi_{r}$ can be taken as diagonal in the above mass formulas Even so, the terms linear in the fields in the scalar and fermion mass matrices will not be diagonal, making the effective potential very difficult to evaluate in general The QSR nature of the theory, however, provides us with a convenient technique for approximatıng the effective potential, because in the absence of soft breakings $V_{1}$

\footnotetext{
* This notation is related to that of ref [1] as follows If $x, 1=-1,2,3$ then $\phi_{\mathrm{x}}=4_{1}, \phi_{\mathrm{x}} \mathrm{z}_{3}=B_{\mathrm{k}}$,

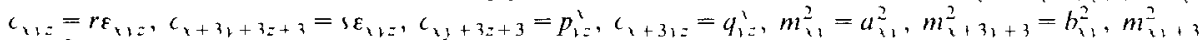

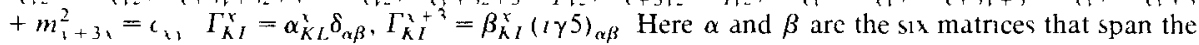
space of real traceless antisymmetric $4 \times 4$ matrices, forming a homomorphism between the fundamental representations of $\mathrm{O}(6)$ and $\mathrm{SU}(4)$ and have an algebra simular to that of the Paulı matrices $\left[\alpha^{x}, \alpha^{\prime}\right]=-2 \varepsilon^{\prime \prime}=\alpha^{z},\left[\beta^{2}, \beta^{1}\right]=-2 \varepsilon^{\prime \prime 2} \beta^{2}\left[\alpha^{2}, \beta^{\prime}\right]=0$
} 
vanıshes, suggestıng we expand the supertrace about the symmetric theory - an expansion that will be valid when the fields are large compared to the massive parameters in the theory

The analysis of the effective potential will demonstrate that for appropriate. but not very restrictive, choices of the parameters, the theory is stable [5] and allows for spontaneous symmetry breakıng via a Coleman-Weinberg-lıke mechanısm

Symbolıcally, writıng (cf eq (25))

$$
M^{2}=m^{2}+T+M^{2}
$$

for either the fermion or scalar mass matrix, with $M^{21}$ the matrix for the supersymmetric theory and $T$ the linear term, we expand in the breakıng terms

$$
\begin{aligned}
\operatorname{Str} M^{4} \ln M^{2} & =\operatorname{Str} M^{4} \ln \left[M^{2 \prime}\left(1+\frac{m^{2}+T}{M^{2 \prime}}\right)\right] \\
& =\operatorname{Str} M^{4} \ln M^{2 \prime}+\operatorname{Str} M^{4} \ln \left(1+\frac{m^{2}+T}{M^{2 \prime}}\right) \\
& =\operatorname{Str} M^{4} \ln M^{2 \prime}+\operatorname{Str}\left(m^{2} M^{2 \prime}+{ }_{2}^{3} T^{2}\right)+\mathrm{O}(\phi)
\end{aligned}
$$

(Note $\operatorname{Tr} M^{2 \prime} T=0=\operatorname{Tr} T M^{2 \prime} \ln M^{2 \prime}$. by symmetry of the matrices)

The second line follows if $\left[m^{2}+T \cdot M^{2 \prime}\right]=0$, which we will now demonstrate Consider eq (24) Because we are working in a flat direction the $M^{2 \prime}$ matrices are diagonal in the group space indices in the adjoint representation. $\left(T^{a}\right)^{h c}=-i f^{\text {ahe }}$. so $f^{u c e} f^{b d t} \phi^{c} \phi^{d}=\left(T^{c} T^{d}\right)_{a b} \phi^{c} \phi^{d}$, lying in the Cartan subalgebra of diagonal matrices By the same reasoning the linear $(T)$ terms are diagonal in group space Then one checks that $\left[T, M^{2 \prime}\right]=0$ in the remaining indices, for the scalar matrix, the totally antisymmetric objects $c_{1, k}$, contracted with $\phi_{h}^{c}$. will either commute past $\delta_{1,}$ or give zero when contracted with another field in $M_{\mathrm{S}}^{2 \prime}$, and for the fermions one finds, using $\alpha^{\prime} \alpha^{\prime}=\beta^{\prime} \beta^{\prime}=-\delta^{\prime \prime}$ and the commutation relations for $\alpha$ and $\beta$ matrices (see previous footnote), that $M_{\mathrm{F}}^{+} M_{\mathrm{F}}^{\prime}$ is proportional to the unit matrix in the $K, L$ indices This last statement also gives $\left[m_{\mathrm{f}}^{2}, M_{\mathrm{F}}^{+} M_{\mathrm{F}}^{\prime}\right]=0$ It is not true in general that $\left[m^{2}, M_{\mathrm{s}}^{2 \prime}\right]=0$, but under the trace the only order $\phi^{2}$ contribution to (26) from this commutator is $\operatorname{Tr} M^{4 \prime}\left[\ln M^{2 \prime},\left(M^{2 \prime}\right)^{-1} m^{2}\right]$, which is zero

Then using $\operatorname{Str} M^{4 \prime} \ln M^{2 \prime}=0$,

$$
V_{\mathrm{eff}}=V_{0}+\frac{1}{64 \pi^{2}} \operatorname{Str}\left[\left(\left\{m^{2} \cdot M^{2 \prime}\right\}+T^{2}\right) \ln \frac{M^{2 \prime}}{\mu^{2}}+\frac{3}{2} T^{2}+m^{2} M^{2 \prime}\right]
$$

This form can also be obtained from evaluatıng directly the sum of one-loop graphs, insertıng up to two breaking vertices in each graph, (but if one absorbs the 
polynomials from the expansion into the counterterms one will be using a different renormalization prescription from eq (23)) The supertrace is over fermions and scalars only An important result of this approximation is that the leading behavior of the effective potential is of order $\phi^{2}$, so the theory appears stable in the directions where the cubic and quartic terms vanish, as long as the signs of the masses are chosen to be positive when the theory is renormalized at large scales The full justification behind this point involves the renormalization group, as described in sect 3

We now search for a minimum of the potential (other than the origin) For simplicity let us examine only the $\phi_{a 3}$ direction, 1 e $\phi_{a l}=\phi_{a} \delta_{t 3}-$ a choice which, because of the explicit breaking of the original $0(6)$ symmetry by the soft-breaking terms, provides a loss of generality, yet which will demonstrate that a global mınımum away from the origin does exist somewhere in field space, for as $V_{\text {eff }}(0)=0$ the fact that $V_{\text {eff }}<0$ for some $\phi$ indicates the existence of such a minımum, provided $V_{\text {eff }} \geqslant 0$ as $\phi$ goes to infinity

In the (flat) $\phi_{u} \delta_{t 3}$ direction, take the directional derivative of eq (27) by multuplying $\phi$ by a scale factor $\kappa$ and finding $\kappa \partial V / \partial \kappa$ Because of homogeneity the derivative on all terms except the logarithm gives us twice the original expression, so If we choose the renormalization point $\mu$ by requirıng that $V_{\mathrm{I}}=0$ at the minımum. $\phi_{\min }$. of the potential, these terms vanish, leaving to

$$
\kappa \frac{\partial V_{\mathrm{eff}}}{\partial \kappa}\left(\phi_{\mathrm{min}}\right)=\left(m^{2}\right)_{33}\left(\kappa \phi^{a}\right)^{2}+\left(\frac{1}{64 \pi^{2}}\right) 2 \operatorname{Str}\left[2 m^{2} M^{2 \prime}+T^{2}\right]
$$

where we have used the fact that $M^{2 \prime}$ is diagonal in the chosen direction, and have assumed for simplicity that the $m^{2}$ masses are diagonal Employing eq (24).

$$
\begin{aligned}
\operatorname{Str}\left[2 m^{2} M^{2 \prime}+T^{2}\right]=C_{2}(G) g^{2}\left[2 \operatorname{Tr} m^{2}-2\left(m^{2}\right)_{33}-8\left(\operatorname{Tr} m_{\mathrm{f}}^{2}+m_{\mathrm{f} 4} m_{\mathrm{f} 3}\right.\right. \\
\left.\left.+m_{\mathrm{f} 2} m_{\mathrm{f} 1}\right)+\frac{1}{g^{2}}\left(c_{i, 3}\right)^{2}\right]\left(\kappa \phi^{a}\right)^{2}
\end{aligned}
$$

Recalling that $\operatorname{Str} M^{4}$ is related to the beta-functions through the renormalization group equation (see eq (31)), it is not surprising that the above factor, which is Str $M^{4}-\operatorname{Str} m^{2}$, can be re-expressed in terms of the beta function for the scalar mass $\left(m^{2}\right)_{33}$ From ref [1] (in the present notation)

$$
\beta_{m_{3,3}^{2}}=\frac{g^{2} C_{2}}{16 \pi^{2}}\left[2 \operatorname{Tr} m^{2}-8\left(\operatorname{Tr} m_{\mathrm{f}}^{2}+m_{\mathrm{f}_{4}} m_{\mathrm{f}_{3}}+m_{\mathrm{f}_{1}} m_{\mathrm{f}_{2}}\right)+\frac{1}{g^{2}} c_{l j 3} c_{i j 3}\right]
$$


and therefore

$$
\frac{1}{64 \pi^{2}} \operatorname{Str}\left[2 m^{2} M^{2 \prime}+T^{2}\right]=\frac{1}{4}\left[\beta_{m_{34}^{2}}-\gamma\left(m^{2}\right)_{33}\right]\left(\kappa \phi^{u}\right)^{2} .
$$

with $\gamma=2 g^{2} C_{2}(G) / 16 \pi^{2}$ The minımum condition (28) becomes

$$
(2-\gamma)\left(m^{2}\right)_{33}+\beta_{m_{3}^{2}}=0
$$

which is the promised relation among masses The term proportional to $\gamma$ is of higher order at the minimum (where $\left(\mathrm{m}^{2}\right)_{33}$ is of order $g^{2}$, so $\gamma\left(\mathrm{m}^{2}\right)_{33}$ is of order $g^{4}$ ) and will be dropped

To see that this is indeed a relative minımum in the direction being considered, we calculate

$$
\begin{aligned}
& V_{\mathrm{eff}}^{\prime \prime}\left(\phi_{\mathrm{m} 1 \mathrm{n}}\right)=\left[\beta_{m_{13}^{\prime}}\right]\left(\phi_{\mathrm{min}}\right)^{2}, \\
& V_{\mathrm{eff}}\left(\phi_{\mathrm{min}}\right)=-{ }_{4}^{1}\left[\beta_{m_{32}^{2}}\right]\left(\phi_{\mathrm{min}}\right)^{2}
\end{aligned}
$$

There is a minımum if $\beta>0$ (whereupon $m_{33}^{2}<0$, as expected) As the relation (2 12) is a restriction only on ratios of masses, and not absolute masses, the scale of the minimum can be chosen larger than any values of the parameters, as required for the approximation to be valid We therefore have a Coleman-Weinberg-like mechanism of dimensional transmutation, with ratios of masses and massive couplings playing the part of dimensionless couplings The relation at the minımum trades in one of the free ratios of masses for one less free ratio and an arbitrary scale, $\phi_{\mathrm{min}}$

\section{The improved effective potential}

In order to know if the relation (2 12) is consistent with a stable theory we need to compare a calculation of the effective potential made at two widely separate scales - the scale of the minımum and an arbitrarily large scale representıng the behavior at infinity The large logarithms which then occur in eq (23) when the fields become large, apparently causing a breakdown of perturbation theory, are controlled by redefining the renormalization scale, $\mu$, leading to a new and renormalization-group-improved effective potential In particular we require that the scalar mass $\left(m^{2}\right)_{32}$ be able to run with scale so that it is positive at large $\phi^{2}$ but becomes negative as it approaches the chosen scale of the minımum

The renormalization group equation obeyed by the exact eftective potential is

$$
\left(\mu \frac{\partial}{\partial \mu}+\beta_{p} \frac{\partial}{\partial \lambda_{p}}-\gamma \phi^{a t} \frac{\partial}{\partial \phi^{a t}}\right) V\left(\phi^{a t}, \lambda_{p}, \mu\right)=0
$$


(there is no gauge parameter in Landau gauge), where $\lambda_{p}$ is the set of masses and massive coupling constants The anomalous dimension, which is the same for all the fields because of the $\mathrm{O}(6)$ symmetry of the unbroken theory, depends only on the gauge coupling $g$, to one loop we have $\gamma=2 g^{2} C_{2} / 16 \pi^{2}$ The solution to this equation is therefore simplified because of the QSR feature $\beta_{g}=0$, so the anomalous dimension is a constant

The equation is solved using Cauchy's method of characteristics The solution can be written as

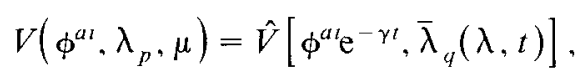

with

$$
\begin{gathered}
t=-\ln \frac{\mu}{\mu_{0}}, \\
\frac{\partial}{\partial t} \bar{\lambda}_{p}\left(\lambda_{q}, t\right)=\beta_{p}\left[\bar{\lambda}_{q}(\lambda, t)\right],
\end{gathered}
$$

where $\hat{V}$ is any function and $\mu_{0}$ is an arbitrary value at which the initial conditions are defined

$$
\begin{aligned}
\bar{\lambda}_{p}(\lambda, 0) & =\lambda_{p}, \\
\hat{V}\left[\phi_{u l}, \bar{\lambda}_{p}(\lambda, 0)\right] & =\hat{V}\left(\phi_{a l}, \lambda_{p}\right)=V\left(\phi_{u t}, \lambda_{p}, \mu_{0}\right)
\end{aligned}
$$

The solution is checked by inserting it into ( 31$)$ and using the deducible equation

$$
\beta_{p}(\lambda) \frac{\partial \bar{\lambda}_{q}}{\partial \lambda_{p}}-\frac{\partial \bar{\lambda}_{q}}{\partial t}=0
$$

For field magnitudes on the order of $\mu_{0}$ we already have a one-loop approximation to $V_{\text {eff }}$. Let $\phi_{0}^{a l}$ be a value of the field at which $V_{\text {eff }}$ is equal to the tree potential

$$
V\left(\phi_{0}^{a l}, \lambda_{p}, \mu_{0}\right)=V_{0}\left(\phi_{0}^{a l}\right)
$$

The choice of $\phi_{0}^{a l}$ selects out a direction in field space We find an expression for the potential at all scales in this particular direction by using dimensional analysis

$$
\begin{aligned}
V\left(\kappa \phi_{0}^{u_{l}}, \lambda_{p}, \mu_{0}\right)=\kappa^{4} V\left(\phi_{0}^{a l}, \lambda_{p} / \kappa, g, \mu_{0} / \kappa\right), \\
\lambda_{p} / \kappa \equiv\left(m_{t j}^{2} / \kappa^{2}, m_{\mathrm{f}} / \kappa, c_{i, k} / \kappa\right) .
\end{aligned}
$$

and then employing the solution (32) at these values of $\phi . \lambda$, and $\mu$, and using the 
fact that $g$ does not run

$$
\begin{array}{r}
V\left(\phi_{0}^{u t}, \lambda_{p} / \kappa, g, \mu_{0} / \kappa\right)=\hat{V}\left[\phi_{0}^{u t} \mathrm{e}^{-\gamma t}, \bar{\lambda}_{p}(\lambda / \kappa, t), g\right] \\
t=-\ln \left(\mu_{0} / \kappa\right) / \mu_{0}=\ln \kappa
\end{array}
$$

So as we scale along this direction the result is

$$
V\left(\kappa \phi_{0}^{a t}, \lambda, \mu_{0}\right)=\mathrm{e}^{4 t} V_{0}\left[\phi_{0}^{u t} \mathrm{e}^{\gamma t}, \bar{\lambda}_{4}\left(\lambda \mathrm{e}^{-t}, t\right), g\right],{ }_{t=\ln \kappa}
$$

As the running couplings are homogeneous in the massive parameters [1] we have.

$$
\bar{\lambda}_{p}(\lambda, \boldsymbol{\kappa}, t)=k^{-\delta_{p}} \bar{\lambda}_{p}(\lambda, t) . \quad \delta_{p}=\text { dimension of } \lambda_{p}=1 \text { or } 2
$$

If $\phi_{0}^{a t}$ is chosen to be a flat direction, the tree potential reduces only to the mass term

$$
\left.V\left(\kappa \phi_{0}^{u l}, \lambda, \mu_{0}\right)={ }_{2}^{1} \mathrm{e}^{(2} \quad 2 \gamma\right) t \bar{m}_{1,}^{2}(\lambda t) \phi_{0}^{u\left(\phi_{0}^{u \prime}\right.}
$$

We now seek a minımum along this direction by differentiatıng with respect to $\kappa$ (equivalently, $t$ ), if ue choose a single $O(6)$ direction, such as $\phi_{11}^{u t}=\phi_{0}^{u} \delta^{\prime 3}$ the arbitrary point $\phi_{0}$ drops out. leaving

$$
2(1-\gamma) \bar{m}_{33}^{2}(\lambda, t)+\beta_{m_{32}^{2}}[\bar{\lambda}(\lambda, t)]=0 .
$$

which at $t=0$ is identical to the previous perturbative result (212) if we choose $\phi_{0}=\phi_{\min }$ (and again drop the higher-order $\gamma$ term) Our interest here is in the behavior of (38) for large fields, $t=\ln \kappa \rightarrow \infty$ Scalıng up from the minimum to large fields one finds the leading behavior of the running masses to be, $\mathrm{eg}$,

$$
\bar{m}_{33}^{2}(t) \sim \frac{1}{48 g^{2}}\left[\left(c_{1, h} ;^{2}+\left(c_{1,3}\right)^{2}\right] \mathrm{e}^{2 h t}, \quad b=\frac{12 g^{2} C_{2}}{16 \pi^{2}}=6 \gamma\right.
$$

and similar equations for the other scalar masses, the coefticient is positive, indicating that for large enough fields all masses will be positive. insuring stability The converse is not true, $1 \mathrm{e}$. not all values of positive masses at large initial scale $t=0$ will run so as to satisfy the minimum condition at some $t<0$ The condition 
(39), in terms of these initial values, is

$$
\begin{aligned}
m_{33}^{2}=\left[\frac{5}{6}\right. & \left.+\left(\gamma+\frac{1}{6}\right) \mathrm{e}^{b t}\right]^{-1} \\
\times & \left\{\left[\left(\gamma+\frac{1}{6}\right) \mathrm{e}^{b t}-\frac{1}{6}\right] \operatorname{Str}^{\prime} m^{2}+\left[\left(b+\frac{1}{2}\right) \mathrm{e}^{2 h t}+\frac{1}{2}-(b+1) \mathrm{e}^{b t}\right]\left(\frac{1}{36 g^{2}}\right)\left(C_{1, h}\right)^{2}\right. \\
& +\left[\left(b+\frac{1}{2}\right) \mathrm{e}^{2 h t}-\frac{1}{2}\right]\left(\frac{1}{12 g^{2}}\right)\left(C_{3 j k}\right)^{2} \\
& \left.+\left[\frac{1}{6}-\frac{1}{6} \mathrm{e}^{h t}+(1+\gamma) b t \mathrm{e}^{h t}\right]\left(\frac{1}{6 g^{2}}\right)\left(C_{1 j k} c(f)_{1, k}\right)\right\},
\end{aligned}
$$

where $\operatorname{Str}^{\prime} m^{2}=\operatorname{Str} m^{2}-m_{33}^{2}$, and $C_{1 j h}$ is related to the initial values of the cubic couplings, $c_{1, k}$, by $C_{1, k}=c_{1, k}-c(f)_{l, k}$, with $c(f)$ the finiteness-condition values [1] At $t=0$ any initial values for the parameters other than $\left(\mathrm{m}^{2}\right)_{33}$ may be chosen, with the allowed values of $\left(\mathrm{m}^{2}\right)_{33}$ that will result in a mınımum being given by letting $t$ on the right-hand side of (310) range over all values $-\infty<t<0$ - with everything subject to the further restriction that the scalar masses be positive at $t=0$, and if $\bar{m}_{33}^{2}(t)$ is less than zero at that value of $t$ (see eq $(213)$ ) These are rather broad restrictions for example, if one chose $C_{l / k}=0$ (finiteness condition for cubic couplings), these conditions can be satısfied for any value of $\operatorname{Str}^{\prime} \mathrm{m}^{2}$ and a range of values of $m_{33}^{2}$ (And we remark again that the necessity that the calculated minımum be at a scale higher than any mass threshold is easily achieved for, roughly, $t=\ln \left(\phi / \phi_{0}\right)$, so if the minimum occurs at $t_{\min }$ we simply select $\phi_{0}$ to be large enough so that $\phi_{\min }$ is larger than any mass at that value of $t$ )

\section{Discussion}

The awkwardness of softly-broken finite models [7] stem from their artificialıty in arranging breakıng parameters to preserve finiteness - this is an unjustifiable extension of the valid concept of arranging fermionic and bosonic particle content to obtain cancellations of graphs The natural minımal extensions of finıte globallysupersymmetric theories are QSR models Because in QSR models the finiteness relations are approached in the infrared regime, the beauty of finite theories, their predictions for mass relations, need not be completely lost The excitement of finite superstring theories has dimınished interest in these kinds of models. since it is assumed that, well below the Planck scale, there remains a softly-broken. $N=1$ globally supersymmetric theory. The primary motivation for insisting on $N=1$ is the desire for manifestly chiral theories However, should interest turn to left-right symmetric models with soft or spontaneous breaking of parity, it is much less clear 
which, if any, type of low-energy supersymmetry will survive In such a context quasi-super-renormalizable models of the kind considered in this and our previous paper may become phenomenologically more attractive

We wish to thank E Rabinovici for extensive discussions and for collaboratıng during the earlier phases of this work This work was supported in part by the US Department of Energy

\section{References}

[1] M B Einhorn G Goldberg and E Rabinovic1, Nucl Phys B256 (1985) 499

[2] C T Hill $\mathrm{m}$ Ann Arbor Workshop on Grand Unfilation (1981) p 258

J Bagger, S Dimopoulos and E Ma»o, Phys Rer Lett 55 (1985) 920)

[3] B Gato, J Leon. J Perez-Mercader and M Quiros Nucl Phy B246 (19x4) 120)

J Ellis, Lectures at the 28th Scottish Universitie, Summer School, CERN-TH-4255, 85 (Edinburgh 19851

[4] S Coleman and E Weinberg, Phys Rer D7 (1973) 1888

[5] S Coleman, $m$ Int School of Subnuclear Physics Ettore Majorana, ed Zichuha (1973)

[6] J M Frere L Mezincescu and Y-P Yao, Phys Rcr D29 (1984) 1196 